\title{
Confirmation that bevacizumab increases the risk of gastrointestinal perforation
}

A meta-analysis of 17 randomized controlled trials of bevacizumab for the treatment of various solid tumors confirms an association with

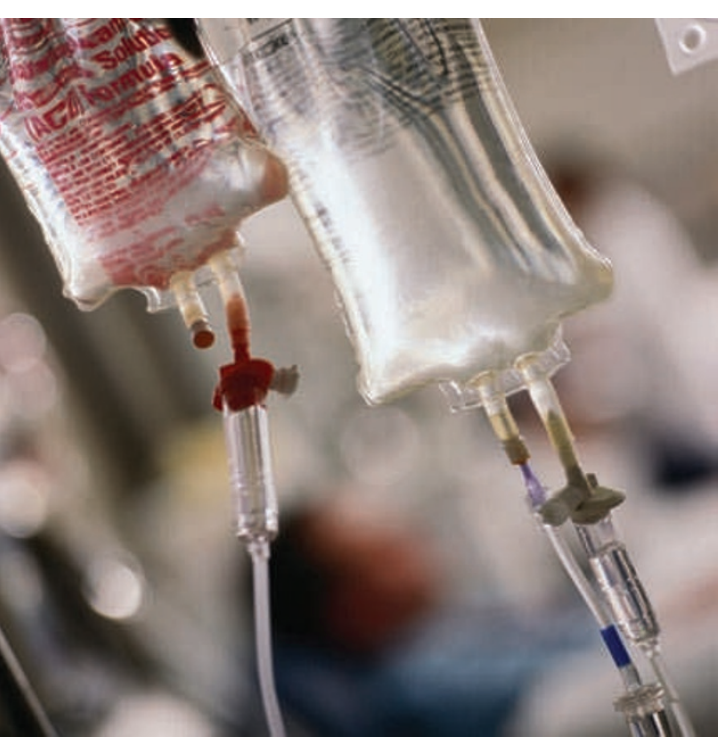

gastrointestinal perforation that led to implementation of a black-box warning by the US FDA. The link between increased risk of gastrointestinal perforation and bevacizumab emerged during two small trials. Until now, however, it was not clear whether these results were a function of confounding factors specific to those studies. "We now show unequivocally that bevacizumab is significantly associated with gastrointestinal perforation," notes senior author Shenhong Wu. Bevacizumab is a recombinant humanized monoclonal antibody against vascular endothelial growth factor; it was the first approved agent to target tumor angiogenesis. The systematic review-which encompassed trials involving 12,294 patients conducted before the 2004 approval-revealed a $0.9 \%$ incidence of gastrointestinal perforation. The overall relative risk in patients treated with bevacizumab compared to control groups was 2.14.
Patients receiving higher doses of the drug, and those being treated for renal cell cancer, were at greatest risk.

"Further studies are now needed to investigate the risk factors that lead to gastrointestinal perforation in cancer patients," suggests Wu. He also recommends a review of the policy that currently excludes patients who have experienced gastrointestinal perforation from further bevacizumab therapy. "If we understand why patients are reacting in this way, selected use of bevacizumab may be possible in some patients who have fully recovered from perforation," Wu concludes.

Kathryn Senior

Original article Hapani, S. et al. Risk of gastrointestinal perforation in patients with cancer treated with bevacizumab: a meta-analysis. Lancet Oncol. 10, 559-568 (2009). 